\title{
Radiofrequency ablation combined with esophageal stent in the treatment of malignant esophageal stenosis: A single-center prospective study
}

\author{
YONGGUI ZHANG ${ }^{1}$, MINGWEI ZHOU ${ }^{2}$, LIN BAI $^{3}$, RONGYAN HAN ${ }^{1}$, KANG LV $^{4}$ and ZHE WANG ${ }^{1}$ \\ Departments of ${ }^{1}$ Gastroenterology, ${ }^{2}$ Dermatology, ${ }^{3}$ Nuclear Medicine and ${ }^{4}$ Emergency, \\ China-Japan Union Hospital of Jilin University, Changchun, Jilin 130033, P.R. China
}

Received January 12, 2018; Accepted June 18, 2018

DOI: $10.3892 / \mathrm{ol} .2018 .9046$

\begin{abstract}
The purpose of this study was to investigate the efficacy of radiofrequency ablation (RFA) combined with esophageal stent in treating malignant esophageal stenosis. Seventy patients with malignant esophageal obstruction treated in Department of Gastroenterology from April 2013 to April 2015 in China-Japan Union Hospital of Jilin University were enrolled. They were randomly assigned into the treatment group (radiofrequency ablation combined with esophageal stent) and control group (esophageal stent). To observe the degree of dysphagia, esophageal stenosis diameter, readmission time, adverse events and complications. There was no significant differences in dysphagia and esophageal diameter between the treatment group and the control group within 1-3 months after operation ( $P>0.05)$, and the degree of dysphagia and esophageal diameter in the treatment group at postoperative 6 months were better than those in the control group $(\mathrm{P}=0.018$ and 0.038 , respectively). The readmission time of the treatment group was also better than that of the control group $(\mathrm{P}=0.021)$. The adverse events and complications included hemorrhage, perforation and esophageal stent displacement. No significant differences in adverse events and complications between the treatment group and the control group were observed. All patients were successfully treated during hospitalization. Effect of radiofrequency ablation combined with esophageal stent implantation was better than esophageal stent implantation in the treatment of malignant esophageal stenosis, but it had no effect on the survival time.
\end{abstract}

Correspondence to: Dr Zhe Wang, Department of Gastroenterology, China-Japan Union Hospital of Jilin University, 126 Xiantai Street, Changchun, Jilin 130033, P.R. China

E-mail: wangzhe8809@163.com

Key words: radiofrequency ablation, esophageal stent, esophageal cancer, esophageal stenosis

\section{Introduction}

Esophageal cancer is one of the most common diseases, it had a very high morbidity and mortality in the world. More than $90 \%$ of esophageal cancer are squamous cell carcinomas. The main clinical manifestations of advanced esophageal cancer are progressive dysphagia. Patients often died of failure to eat accompanied by organ failure. At present, the commonly used treatment methods include local surgical resection and palliative treatment. Local surgical excision has a large injury, patients often cannot tolerate. Common palliative treatment methods include endoscopic dilatation, placement of esophageal stents, endoscopic chemical therapy for slow-release particle implantation and nasogastric feeding (1). In 1983, Frimberger first applied metal stents in the treatment of patients with esophageal stenosis (2), the method has been gradually recognized and widely used $(3,4)$. Some scholars believed that stent implantation should be the first choice for inoperative malignant stenosis, which is currently an effective method to relieve dysphagia caused by advanced esophageal cancer (5-7). Radiofrequency ablation, as an approach of treating malignant tumors, was primarily used in the treatment of tumors in hollow viscera, such as biliary tumors and colorectal cancers (8-11). Its working mechanism is mainly through thermal damage on tumor tissue, thus destroying tumor tissues. Radiofrequency ablation in the treatment of esophageal stenosis is still rare. This study was designed to explore the radiofrequency ablation combined with esophageal stent in the treatment of advanced esophageal stenosis. Therefore, a single-center prospective study of radiofrequency ablation combined with esophageal stent was designed for treatment of malignant esophageal stenosis.

\section{Patients and methods}

Patients. A total of 70 patients with malignant esophageal obstruction undergoing gastroenterology in our hospital from April 2013 to April 2015 were enrolled, including 36 males and 34 females, aged from 43 to 82 years, with an average of 64.4 years. Inclusion criteria: i) Advanced esophageal squamous cell carcinoma confirmed by ugi, enhanced CT scan of the chest, gastroscopy and biopsy, abdominal and 
neck ultrasound. ii) The tumor was located in the thoracic esophagus. iii) Expected survival time $>3$ months before stenting. iv) No esophageal cancer radical indications. v) In addition to stent placement treatment, follow the patient's subjective intention, radiotherapy and chemotherapy were not performed. Exclusion criteria: i) Advanced esophageal cancer patients with cachexia, coma and give up treatments. ii) Perforation of the esophagus caused by esophageal fistula and other causes of cancer. This study was approved by the Ethics Committee of China-Japan Union Hospital of Jilin University (Jilin, China). Patients and their families signed an informed consent form before treatment.

Methods. Included patients in the study were randomly assigned to the treatment group (radiofrequency ablation combined with esophageal stent) and control group (esophageal stent), each group included 35 patients. In the treatment group, 19 were males and 16 were females, aged $43-79$ years, the mean age was 63.5 years. In the control group, 17 were males and 18 were females, aged 52-82 years, the mean age was 64.9 years. Procedure in the treatment group was: i) Under the guidance of digital subtraction angiography (DSA), the guide wire was inserted through the esophageal stenosis, the endoscope was placed over the obstruction site through the oral cavity, and the radiofrequency ablation catheter was inserted through the working channel, 2 electrodes of the radiofrequency ablation catheter were in touch with the tumor surface under the endoscope, circumferential radiofrequency ablation therapy was then performed. ii) Retain the guide wire, stent was placed into the lesions guided by the guide wire. Release the stent according to the marking position so that the upper and lower edges of the stent were exceeded the lesion by $2.0 \mathrm{~cm}$. After releasing the stent, the carrier and guidewire was taken out. Procedure in the control group was the second step in the treatment group guided with DSA. The main equipment: Habib EndoHPB RFA endoscope (EMcision, London, UK), Olympus GIF-Q260J endoscopy (Olympus, Tokyo, Japan) esophageal stent coated with silicone (N-SE-G-20/100-A-8/650).

Complication management. Intraoperative hemorrhage patients in the treatment group were treated with local injection of norepinephrine for hemostasis under endoscopy, and then esophageal stent implantation was performed. Intraoperative perforation patients were given to the covered stent to block the perforation site. Patients with intraoperative stent displacement achieved the predicted position after adjusting the stent by placing forceps into the working channel under endoscope. All patients successfully completed the treatment, no major complications which needed surgically treatment were occurred.

Efficacy evaluation. According to the evaluating standards of WHO and Stooler classification of dysphagia and esophageal stenosis (12): Grade 0 means be able to eat normal diet, grade 1 means be able to eat soft rice, grade 2 means be able to eat semi-liquid, grade 3 means be able to eat the liquid, grade 4 means inability to eat or inflow. The degree of esophageal stenosis before and after treatment was measured by upper gastrointestinal angiography. When the contrast medium passed through the stenosis, the stenosis diameter
Table I. Basic characteristics of patients.

\begin{tabular}{lccc}
\hline Characteristics & $\begin{array}{c}\text { Treatment } \\
\text { group }\end{array}$ & $\begin{array}{c}\text { Control } \\
\text { group }\end{array}$ & P-value \\
\hline Sex & & & 0.632 \\
$\quad$ Male & 19 & 17 & \\
Female & 16 & 18 & \\
Esophageal stenosis & & & \\
diameter (mm) & & & \\
2 & 5 & 3 & 0.452 \\
3 & 12 & 10 & 0.607 \\
4 & 8 & 11 & 0.420 \\
5 & 6 & 8 & 0.550 \\
6 & 4 & 3 & 0.690 \\
Section & & & \\
Neck & 0 & 0 & \\
Upper chest & 7 & 4 & 0.324 \\
Middle chest & 20 & 22 & 0.626 \\
Lower chest & 8 & 9 & 0.780 \\
\hline
\end{tabular}

Table II. Postoperative dysphagia degree comparison between the treatment group and control group according to Stooler classification.

\begin{tabular}{lccc}
\hline Grades & $\begin{array}{c}\text { Treatment } \\
\text { group }\end{array}$ & $\begin{array}{c}\text { Control } \\
\text { group }\end{array}$ & P-value \\
\hline $\begin{array}{l}\text { Before the surgery } \\
\text { Grade 0, 1, }\end{array}$ & 12 & 8 & 0.289 \\
$\quad$ Grade 3, 4 & 23 & 27 & \\
Postoperative 1 month & & & 1 \\
$\quad$ Grade 0, 1, & 35 & 35 & \\
$\quad$ Grade 3, 4 & 0 & 0 & \\
Postoperative 3 months & & & 0.643 \\
$\quad$ Grade 0, 1, 2 & 33 & 32 & \\
$\quad$ Grade 3, 4 & 2 & 3 & \\
Postoperative 6 months & & & 0.018 \\
$\quad$ Grade 0, 1, 2 & 29 & 20 & \\
Grade 3, 4 & 6 & 15 & \\
\hline
\end{tabular}

was measured and recorded. The time of inability to eat after the operation was recorded. All patients were followed-up for 24 months. Improvement of dysphagia at 1, 3, and 6 months after surgery was followed-up in Outpatient Department. The stenosis of esophageal stenosis and the incidence of complications were measured by upper gastrointestinal angiography.

Statistical analysis. We used Statistical Product and Service Solutions (SPSS) 16.0 software (SPSS, Inc., Chicago, IL, USA) for statistical analysis. Measurement data of normal distribution were expressed as mean \pm standard deviation (mean $\pm \mathrm{SD}$ ), two independent samples were compared using t-test. Regarding the comparision of the readmission time 

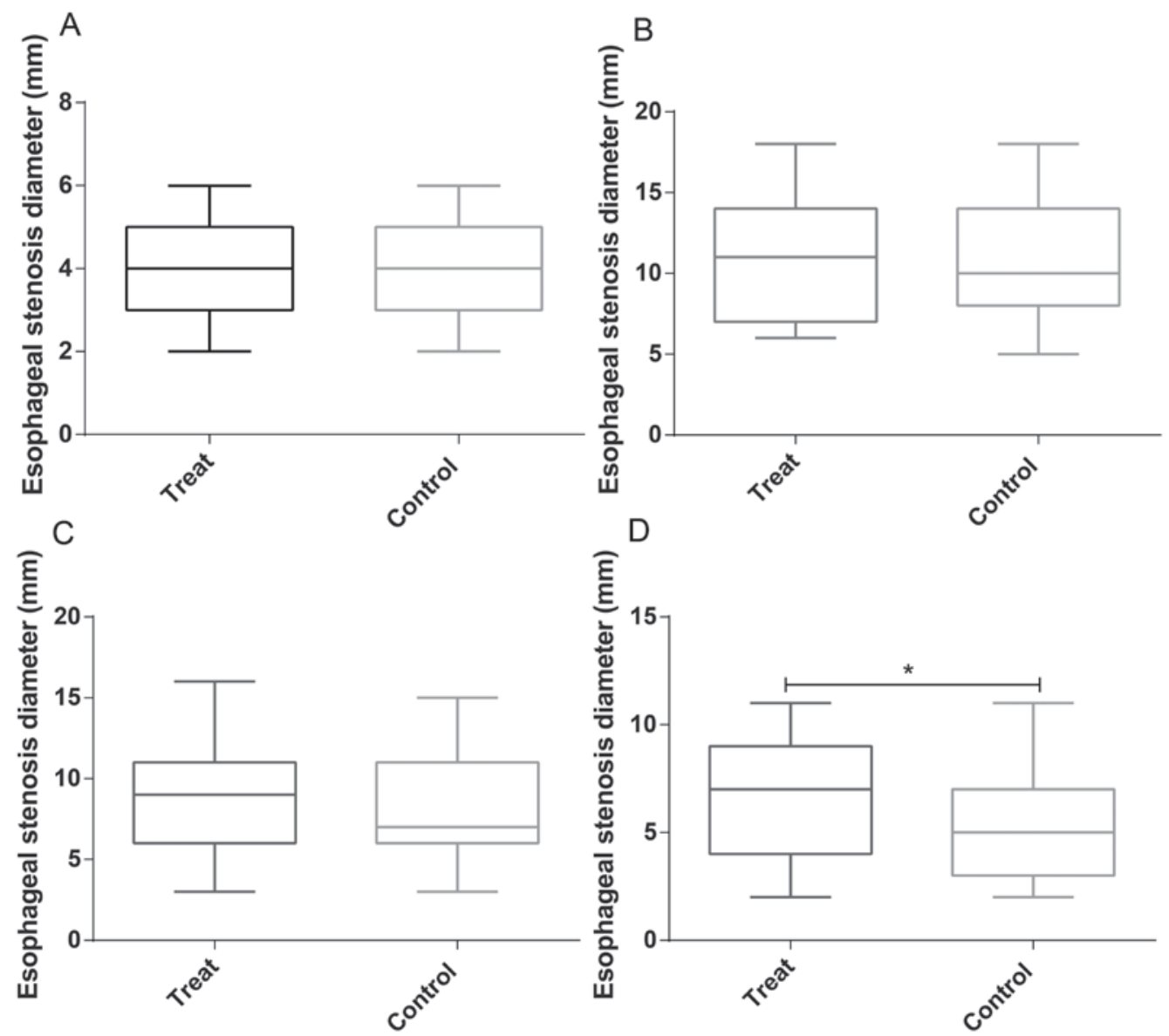

Figure 1. Comparison of esophageal stenosis diameter between the treatment and control groups. (A) There was no significant difference in the esophageal stenosis diameter between the treatment and control groups. (B) One month after treatment, there was no significant difference in the degree of esophageal stenosis between the treatment and control groups. (C) Three months after treatment, there was no significant difference in the degree of esophageal stenosis between the treatment and control groups. (D) Six months after treatment, esophageal stenosis diameter in the treatment group was longer than that in the control group $\left({ }^{*} \mathrm{P}<0.05\right)$.

in treat and control groups, Student's t-test was employed. Counting data were expressed as the number of cases and percentage, comparison of them was analyzed with Chi-square test or rank sum test. $\mathrm{P}<0.05$ was considered to indicate a statistically significant difference.

\section{Results}

Patient characteristics. In the treatment group, 19 were males and 16 were females, aged 43-79 years, the mean age was 63.5 years old. In the control group, 17 were males and 18 were females, aged 52-82 years, the average age was 64.9 years old. There was no significant difference in sex, pre-treatment esophageal stenosis and esophageal stenosis $(\mathrm{P}>0.05)$. Results were listed in Table I.

Comparison of dysphagia degree. In the present study, no significant difference in preoperative dysphagia degree between the treatment group and the control group was found $(\mathrm{P}>0.05)$. One month after treatment, the relief rate of dysphagia in both groups was the same. Three months after treatment, no significant difference in the dysphagia degree was observed between the two groups $(\mathrm{P}>0.05)$. However, dysphagia degree in the treatment group was better than that in the control group 6 months after the treatment. Patients whose Stooler grade was 0-2 in the treatment group were more than the control group, the difference was statistically significant $(\mathrm{P}=0.018$ and $\mathrm{P}<0.05)$. Detailed results were shown in Table II.

Comparison of postoperative esophageal stenosis. In our report, we performed gastroscopy to evaluate the degree of esophageal stenosis before treatment and at 1,3 and 6 months after treatment. The results illustrated that no significant difference in esophageal stenosis was exerted between the two groups before treatment $(\mathrm{P}=0.544)$, the improvement of esophageal stenosis 1 and 3 months after treatment in the treatment group was better than that in the control group, but the difference was not statistically significant $(\mathrm{P}=0.730$ and 0.393 , respectively). However, the degree of esophageal stenosis 6 months after treatment improved better in the treatment group in comparison with those in the control group, the difference was statistically significant $(\mathrm{P}=0.038)$. Results were illustrated in Fig. 1.

Adverse events. In this study, no deaths during hospitalization occurred in all enrolled patients. The treatment group had 6 cases of bleeding, and the control group had 7, local spray of norepinephrine for hemostasis was performed. There were 


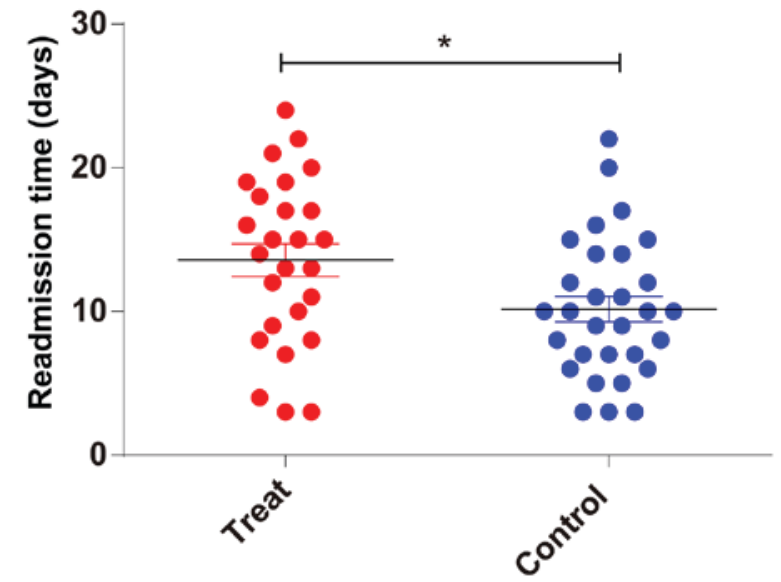

Figure 2. Readmission time in the treatment group was better than that in the control group $\left({ }^{*} \mathrm{P}<0.05\right)$.

Table III. Adverse events of the enrolled patients in treatment and control groups.

\begin{tabular}{lccc}
\hline & $\begin{array}{c}\text { Treatment } \\
\text { group }\end{array}$ & $\begin{array}{c}\text { Control } \\
\text { group }\end{array}$ & P-value \\
\hline Hemorrhage & 6 & 7 & 0.758 \\
Perforation & 2 & 1 & 0.556 \\
Stent displacement & 3 & 2 & 0.643 \\
\hline
\end{tabular}

2 cases of esophageal perforation in the treatment group and 1 in the control group, the perforation was $<5 \mathrm{~mm}$. Perforation was covered with the stent to block the wounds, and these patients were not sent for surgical treatment. Three patients occurred stent displacement occurred in the treatment group and 2 in the control group. There were no significant differences between the two groups in all the above adverse events $(\mathrm{P}>0.05)$. Specific information was listed in Table III.

Prognosis. During the 2 years of follow-up, 12 patients in the treatment group died, with a mortality rate of $34.3 \%$. Seventeen patients in the control group died, with a mortality rate of $48.6 \%$. No significant difference in mortality was found $(\mathrm{P}=0.225$ and $\mathrm{P}>0.05)$. After treatment, the patient was readmitted to the hospital again because of the inability to eat, and the Stooler was graded 4. Readmission in the treatment group was superior to that in the control group, the difference was statistically significant $(\mathrm{P}=0.021)$. The above results were demonstrated in Fig. 2.

\section{Discussion}

The 5-year survival rate of esophageal cancer is only $15-25 \%$ (12). An important problem faced by patients with advanced esophageal cancer is dysphagia resulted from malignant esophageal obstruction. Unable to eat brings great suffering to patients, inadequate nutrient intake and malignant tumor consumption gradually lead to organ failure and even death in patients. Esophageal stent was originally developed to address the surgical treatment of esophageal cancer stenosis, reconstruct the channel, solve patient's nutritional problems and improve the life quality of patients with advanced esophageal cancer. With the development of stent technology, development of covered stent, esophageal stent has been more and more applied in various esophageal fistulas, such as esophageal mediastinal fistula, esophageal tracheal fistula, etc., and achieved good effect (13-15).

Radiofrequency ablation technology is an expanding field in clinical application. In 1990, McGahan et al (16) and Rossi et al (17) first proposed the application of radioactive ablation inactivation to treat cancer patients. In recent years, radiofrequency ablation was gradually applied to digestive tract cancer, its mechanism was emitting high-frequency radio waves through electrodes, stimulating plasma shock of cells and tissues to generate $80-120^{\circ} \mathrm{C}$ heat, rapidly to form tissue gasification and finally, tumor tissues were solidified and necrosis. A reaction zone around the necrosis tumor cut off the blood supply of the tumor to prevent tumor growth and metastasis.

All the 70 patients included in this study successfully completed the corresponding treatment. The success rate of operation was $100 \%$. No serious complications occurred during the operation. In the treatment group, there were 6 cases of hemorrhage, the incidence was $17.1 \%, 2$ cases of perforation, the incidence was $5.7 \%, 3$ cases of stent displacement, the incidence rate was $8.6 \%$, these patients were all improved after conservative treatment. In the control group, there were 7 cases of hemorrhage, the incidence rate was $20.0 \%, 1$ case of perforation, the incidence was $2.9 \%, 2$ cases of stent displacement, the incidence was $8.6 \%$, these patients were all improved after conservative treatment. No significant difference in the incidence of complications was found between the two groups. Although no serious complication occurred in this study, endoscopists still need to pay attention to possible complications associated with radiofrequency ablation, including esophageal perforation, vascular injury, esophageal leakage, esophageal leakage, and sepsis. Among them, the most dangerous and the most common one is the iatrogenic heat injury of the surrounding tissues by radiofrequency ablation, mainly manifested as esophageal perforation and vascular injury.

For exploring the effectiveness of radiofrequency ablation combined with esophageal stent implantation in the treatment of malignant esophageal stenosis, 35 patients with esophageal cancer who underwent esophageal stent placement alone were enrolled in this study as the control group. Followed-up for 2 years, the observation time was postoperative 1, 3 and 6 months, patient's symptoms and esophageal stenosis were accessed. Results showed that within 1-3 months after surgery, there was no difference in the effect between the radiofrequency ablation combined with esophageal stent and esophageal stent alone in treatment of malignant esophageal stenosis $(\mathrm{P}>0.05)$. However, in long-term efficacy over 6 months, radiofrequency ablation combined with esophageal stent was superior to esophageal stent alone in the treatment of malignant esophageal stricture $(\mathrm{P}<0.05)$. In addition, readmission time due to dysphagia in the treatment group was significantly longer than the control group, the difference was statistically significant $(\mathrm{P}<0.05)$.

It is worth mentioning that esophageal stent for malignant esophageal stenosis caused by dysphagia and the treatment of esophageal fistula is safe and effective. There are metal and 
plastic stents. However, they are only served as a palliative treatment to improve the life quality of patients with advanced stage. There have been no reports that significantly prolonged the survival of patients (18-20). Two-year follow-up was conducted in this study, mortality of radiofrequency ablation combined with esophageal stent and esophageal stent alone was similar, the difference was not statistically significant, suggesting that it could not prolong the survival time of patients.

In conclusion, the short-term efficacy of radiofrequency ablation combined with esophageal stent treatment was similar to that of esophageal stent alone. However, the long-term efficacy of which was superior to esophageal stent alone, it prolonged the readmission time, but no influences were observed in the survival time. Limited to the small sample of this study, single-center study, radiofrequency ablation combined with esophageal stent in the treatment of malignant esophageal stenosis still need prospective randomized controlled trials with a large sample to verify.

\section{Acknowledgements}

Not applicable.

\section{Funding}

No funding was received.

\section{Availability of data and materials}

All data generated or analyzed during this study are included in this published article.

\section{Authors' contributions}

YZ was for study design, $\mathrm{MZ}$ and $\mathrm{KL}$ were for data collection and analysis. YZ and LB were for preparation of manuscript, $\mathrm{RH}$ was for literature search, ZW was for funds collection. All authors read and approved the final manuscript.

\section{Ethics approval and consent to participate}

This study was approved by the Animal Ethics Committee of China-Japan Union Hospital of Jilin University (Jilin, China). Signed written informed consents were obtained from the patients and/or guardians.

\section{Patient consent for publication}

Not applicable.

\section{Competing interests}

The authors declare that they have no competing interests.

\section{References}

1. Katsanos K, Sabharwal T and Adam A: Stenting of the lower gastrointestinal tract: Current status. Cardiovasc Intervent Radiol 34: 462-473, 2011.

2. Frimberger E: Endoscopic treatment of benign esophageal stricture. Endoscopy 15 (Suppl 1): 199-202, 1983.
3. Sheikh RA and Trudeau WL: Expandable metallic stent placement in patients with benign esophageal strictures: Results of long-term follow-up. Gastrointest Endosc 48: 227-229, 1998.

4. Zelenák K, Mistuna D, Lúcan J and Polácek H: Broken esophageal stent successfully treated by interventional radiology technique. Cardiovasc Intervent Radiol 33: 643-645, 2010.

5. Kawai T, Miyazaki I, Yagi K, Kataoka M, Kawakami K, Yamagishi T, Sofuni A, Itoi T, Moriyasu F, Osaka Y, et al: Comparison of the effects on cardiopulmonary function of ultrathin transnasal versus normal diameter transoral esophagogastroduodenoscopy in Japan. Hepatogastroenterology 54: 770-774, 2007.

6. Allum WH, Griffin SM, Watson A and Colin-Jones D; Association of Upper Gastrointestinal Surgeons of Great Britain and Ireland; British Society of Gastroenterology; British Association of Surgical Oncology: Guidelines for the management of oesophageal and gastric cancer. Gut 50 (Suppl 5): v1-v23, 2002.

7. Shin JH, Song HY, Ko GY, Lim JO, Yoon HK and Sung KB: Esophagorespiratory fistula: Long-term results of palliative treatment with covered expandable metallic stents in 61 patients. Radiology 232: 252-259, 2004.

8. Valle J, Wasan H, Palmer DH, Cunningham D, Anthoney A, Maraveyas A, Madhusudan S, Iveson T, Hughes S, Pereira SP, et al; ABC-02 Trial Investigators: Cisplatin plus gemcitabine versus gemcitabine for biliary tract cancer. N Engl J Med 362: 1273-1281, 2010.

9. Moss AC, Morris E, Leyden J and MacMathuna P: Malignant distal biliary obstruction: A systematic review and meta-analysis of endoscopic and surgical bypass results. Cancer Treat Rev 33: 213-221, 2007.

10. Soderlund $C$ and Linder $S$ : Covered metal versus plastic stents for malignant common bile duct stenosis: A prospective, randomized, controlled trial. Gastrointest Endosc 63: 986-995, 2006.

11. Ohhigashi S, Nishio T, Watanabe F and Matsusako M: Experience with radiofrequency ablation in the treatment of pelvic recurrence in rectal cancer: Report of two cases. Dis Colon Rectum 44: 741-745, 2001.

12. Pennathur A, Gibson MK, Jobe BA and Luketich JD: Oesophageal carcinoma. Lancet 381: 400-412, 2013.

13. Lee S, Osugi $\mathrm{H}$, Tokuhara $\mathrm{T}$, Takemura M, Kaneko $\mathrm{M}$, Tanaka Y, Fujiwara Y, Nishizawa S, Iwasaki H and Suehiro S: Self-expandable metallic stent for unresectable malignant strictures in the esophagus and cardia. Jpn J Thorac Cardiovasc Surg 53: 470-476, 2005

14. Maroju NK, Anbalagan P, Kate V and Ananthakrishnan N: Improvement in dysphagia and quality of life with self-expanding metallic stents in malignant esophageal strictures. Indian $\mathbf{J}$ Gastroenterol 25: 62-65, 2006.

15. Shim CS, Jung IS, Cheon YK, Ryu CB, Hong SJ, Kim JO, Cho JY, Lee JS, Lee MS and Kim BS: Management of malignant stricture of the esophagogastric junction with a newly designed self-expanding metal stent with an antireflux mechanism. Endoscopy 37: 335-339, 2005.

16. McGahan JP, Browning PD, Brock JM and Tesluk H: Hepatic ablation using radiofrequency electrocautery. Invest Radiol 25: 267-270, 1990.

17. Rossi S, Buscarini E, Garbagnati F, Di Stasi M, Quaretti P, Rago M, Zangrandi A, Andreola S, Silverman D and Buscarini L: Percutaneous treatment of small hepatic tumors by an expandable RF needle electrode. AJR Am J Roentgenol 170: 1015-1022, 1998.

18. Yajima K, Kanda T, Nakagawa S, Kaneko K, Kosugi S, Ohashi M and Hatakeyama K: Self-expandable metallic stents for palliation of malignant esophageal obstruction: Special reference to quality of life and survival of patients. Dis Esophagus 17: 71-75, 2004.

19. Xinopoulos D, Dimitroulopoulos D, Moschandrea I, Skordilis P, Bazinis A, Kontis M, Paraskevas I, Kouroumalis E and Paraskevas E: Natural course of inoperable esophageal cancer treated with metallic expandable stents: Quality of life and cost-effectiveness analysis. J Gastroenterol Hepatol 19: 1397-1402, 2004.

20. Xinopoulos D, Dimitroulopoulos D, Tsamakidis K, Korkolis D, Fotopoulou A, Bazinis A, Kontis M, Vasilopoulos P and Paraskevas E: Palliative treatment of advanced esophageal cancer with metal-covered expandable stents. A cost-effectiveness and quality of life study. J BUON 10: 523-528, 2005.

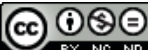

This work is licensed under a Creative Commons Attribution-NonCommercial-NoDerivatives International (CC BY-NC-ND 4.0) License. 